\title{
Molecular Outflows in Galaxy Merger Simulations with Embedded Active Galactic Nuclei
}

\section{Citation}

Narayanan, Desika, Thomas J. Cox, Brant Robertson, Romeel Davé, Tiziana Di Matteo, Lars Hernquist, Philip Hopkins, Craig Kulesa, and Christopher K. Walker. 2006. "Molecular Outflows in Galaxy Merger Simulations with Embedded Active Galactic Nuclei." The Astrophysical Journal 642 (2): L107-10. https://doi.org/10.1086/504846.

\section{Permanent link}

http://nrs.harvard.edu/urn-3:HUL.InstRepos:41381687

\section{Terms of Use}

This article was downloaded from Harvard University's DASH repository, and is made available under the terms and conditions applicable to Other Posted Material, as set forth at http:// nrs.harvard.edu/urn-3:HUL.InstRepos:dash.current.terms-of-use\#LAA

\section{Share Your Story}

The Harvard community has made this article openly available. Please share how this access benefits you. Submit a story.

Accessibility 


\title{
MOLECULAR OUTFLOWS IN GALAXY MERGER SIMULATIONS WITH EMBEDDED ACTIVE GALACTIC NUCLE

\author{
Desika Narayanan, ${ }^{1}$ Thomas J. Cox ${ }^{2}$ Brant Robertson, ${ }^{2}$ Romeel Davé, ${ }^{1}$ Tiziana Di Matteo, ${ }^{3}$ Lars Hernquist, $^{2}$ \\ Philip Hopkins, ${ }^{2}$ Craig Kulesa, ${ }^{1}$ And Christopher K. Walker ${ }^{1}$ \\ Received 2006 March 8; accepted 2006 April 3; published 2006 April 24
}

\begin{abstract}
We study the effects of feedback from active galactic nuclei (AGNs) on emission from molecular gas in galaxy mergers by combining hydrodynamic simulations that include black holes with a three-dimensional, non-local thermodynamic equilibrium (LTE) radiative transfer code. We find that molecular clouds entrained in AGN winds produce an extended CO morphology with significant off-nuclear emission, which may be detectable via contour mapping. Furthermore, kinematic signatures of these molecular outflows are visible in emission-line profiles when the outflow has a large line-of-sight velocity. Our results can help interpret current and upcoming observations of luminous infrared galaxies, as well as provide a detailed test of subresolution prescriptions for supermassive black hole growth in galaxy-scale hydrodynamic simulations.
\end{abstract}

Subject headings: cosmology: theory — galaxies: active — galaxies: formation — galaxies: interactions — galaxies: ISM - line: formation

\section{INTRODUCTION}

The physical processes giving rise to the birth and sustained fueling of massive starbursts and active galactic nuclei (AGNs) have been of interest since their discovery. It is generally agreed that AGNs are powered by accretion of gas onto supermassive black holes (BHs) in the centers of galaxies (e.g., Lynden-Bell 1969), but the fueling mechanism is less clear. Hydrodynamic simulations have shown that mergers can produce strong, galaxy-scale inflows owing to gravitational torques (Barnes \& Hernquist 1991, 1996), triggering starbursts (Mihos \& Hernquist 1996). This suggests a circumstantial link between starbursts and AGN activity.

Observational evidence suggesting the evolution of starbursts into AGNs through mergers is compelling. Some ultraluminous infrared galaxies (ULIRGs; $L_{\mathrm{IR}} \geq 10^{12} L_{\odot}$ ) exhibit spectral energy distributions (SEDs) characteristic of classical starbursts, whereas others have SEDs more closely resembling optical quasars (e.g., Farrah et al. 2003). In seminal papers, Soifer et al. (1987) and Sanders et al. (1988a, 1988b) used optical, near-infrared and millimeter-wave observations to advance a scenario in which starburst-dominated ULIRGs served as antecedents of AGNs. The specifics of a starburst-AGN connection, however, remain under heavy debate throughout the literature (e.g., Sanders \& Mirabel 1996).

Recent numerical models by Di Matteo et al. (2005), Hopkins et al. (2005a, 2005b, 2005c, 2006a), and Springel et al. (2005a) have provided a theoretical foundation for the link between starbursts and AGNs. In particular, by modeling the growth of (and feedback from) central BHs, they showed that gas-rich galaxy mergers are a viable candidate to serve as a precursor to the formation of quasars. Their simulations also show that feedback from accreting BHs in galaxies is relevant to a wide range of phenomena associated with the evolution of galaxies in mergers, including characteristic X-ray emission patterns (Cox et al. 2006), observed quasar luminosity functions and lifetimes (Hop-

\footnotetext{
${ }^{1}$ Steward Observatory, University of Arizona, 933 North Cherry Avenue, Tucson, AZ 85721; dnarayanan@as.arizona.edu.

${ }^{2}$ Harvard-Smithsonian Center for Astrophysics, 60 Garden Street, Cambridge, MA 02138.

${ }^{3}$ Carnegie Mellon University, Department of Physics, 5000 Forbes Avenue, Pittsburgh, PA 15213.
}

kins et al. 2005c), the $M_{\mathrm{BH}^{-}} \sigma$ relation (Di Matteo et al. 2005; Robertson et al. 2006b), the fundamental plane of elliptical galaxies (Robertson et al. 2006a), and the bimodal galaxy color distribution (Springel et al. 2005b; Hopkins et al. 2006b).

There has been a long-standing interest in better understanding the nature of the molecular interstellar medium (ISM) in mergers, as the molecular gas serves as fuel for the induced starburst activity and possibly for accreting $\mathrm{BH}(\mathrm{s})$. High-resolution observations have identified massive concentrations of molecular gas in the nuclear regions of ULIRGs (Bryant \& Scoville 1999), as well as high-excitation molecular gas in regions of massive starbursts (Iono et al. 2004; Wang et al. 2004).

Large surveys at submillimeter and millimeter wavelengths have shown that mergers in the local universe emit copious molecular line radiation both from diffuse molecular gas (e.g., Sanders et al. 1991, among others) and dense cloud cores (e.g., Gao \& Solomon 2004; Narayanan et al. 2005). Other work demonstrates that high- $z$ infrared luminous and submillimeter selected sources also contain significant amounts of molecular gas (Greve et al. 2005; Tacconi et al. 2006). Moreover, as evidenced by IR and X-ray studies, a large fraction of these galaxies at high- $z$ contain AGNs (e.g., Alexander et al. 2005; Polletta et al. 2006). However, despite the wealth of data on molecular emission in interacting galaxies, little is known about the impact of embedded AGNs on this radiation.

Here we describe preliminary attempts to quantify the observable effects of AGN feedback on molecular line emission from major galaxy mergers. We use hydrodynamic simulations of mergers with and without AGNs, combined with a new threedimensional non-LTE radiative transfer code (Narayanan et al. 2006; D. Narayanan et al. 2006, in preparation) to model CO emission. In this Letter, we describe results in which we find distinct signatures of AGN feedback on cold gas, and we discuss some observational results that may be understood in this context.

\section{NUMERICAL SIMULATIONS}

Our hydrodynamic simulations were performed using the $\mathrm{N}$ body/smoothed particle hydrodynamics (SPH) code GADGET2 (Springel 2005). This code uses a fully conservative formulation of SPH (Springel \& Hernquist 2002) and accounts for radiative cooling of the gas (Davé et al. 1999), a multiphase 

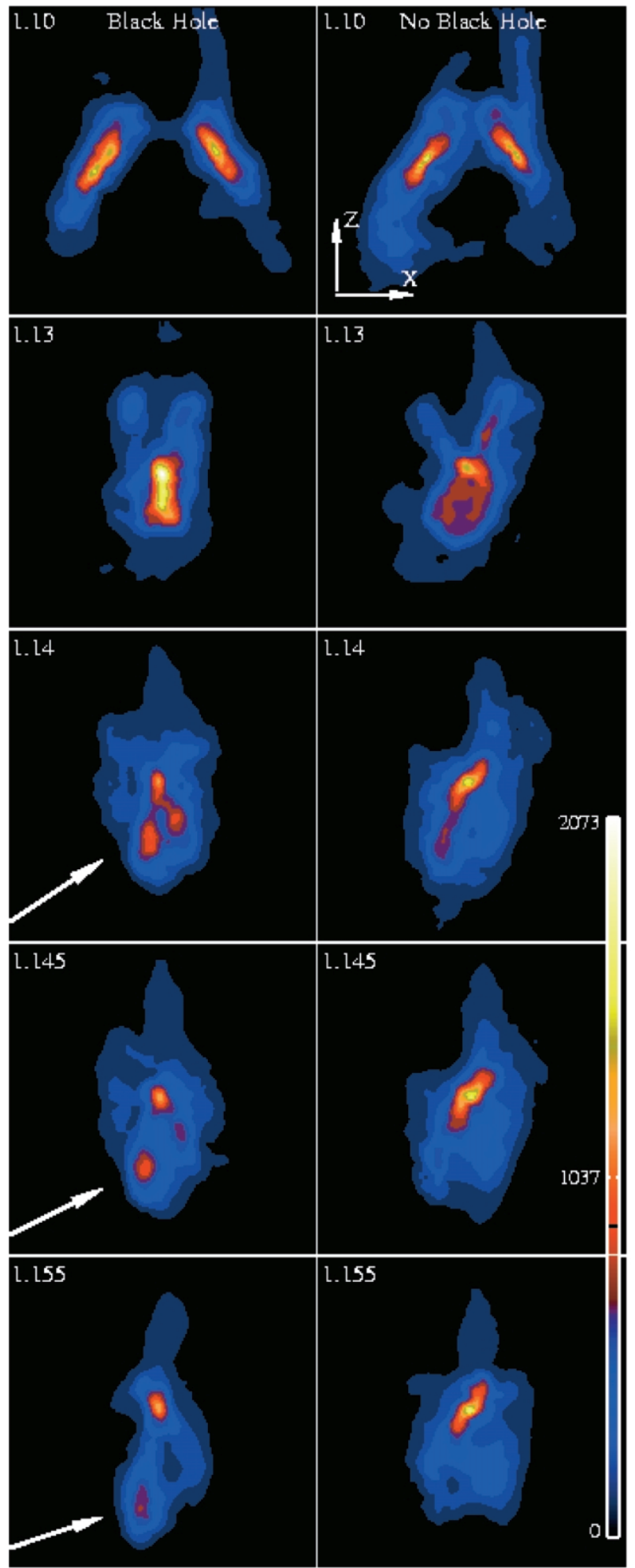

FIG. 1.- $\mathrm{CO} J=1-0$ emission intensity contours for an equal-mass galaxy merger. The left column is a time sequence for the model with BHs, and the right column is without BHs. In the model with BHs, after the galaxies merge, massive amounts of gas are driven into the nuclear regions and accrete onto the supermassive $\mathrm{BH}$. The subsequent AGN feedback energy can blow large blobs of molecular gas out from the nuclear regions (see arrows). These features are not seen in the model without BHs. The time stamp of the image is in the top left of each panel and is in units of $h^{-1}$ Gyr. The color contours are in units of $\mathrm{K} \mathrm{km} \mathrm{s}^{-1}$ (velocity-integrated Rayleigh-Jeans temperature), and the scale is on the right side of the plot. Each panel is $12 h^{-1} \mathrm{kpc}$ on a side. The images are made at $1 / 4 h^{-1} \mathrm{kpc}$ spatial resolution. The coordinate system for use with Fig. 2 is in the top right panel.
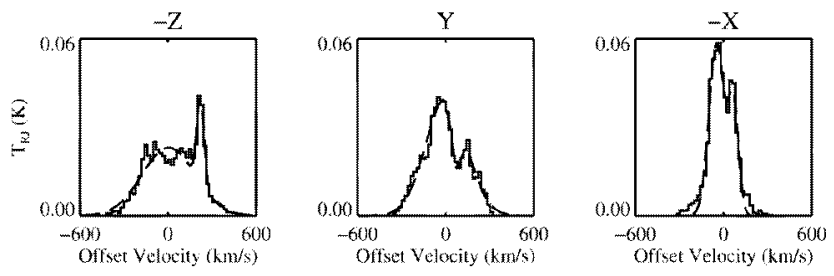

FIG. 2.-Synthetic CO $J=1-0$ (rest frame) emission-line profile taken at $T=1.155 h^{-1} \mathrm{Gyr}$ in the merger run with BHs over three orthogonal viewing angles. The direction of each LOS is above the panels and corresponds to the coordinate system in the top right panel of Fig. 1. The high-velocity peak in the leftmost panel is due to the outflowing gas viewed with a large LOS velocity component. In the $\hat{y}$ direction, the outflow has a smaller LOS velocity, and the emission owing to the outflow blends more into the main emission. In the $-\hat{x}$ viewing angle, outflows have a negligible effect on emission line. The best fits to the model spectra are overplotted with dashed lines. The spectra are taken such that the object is at $z=2$ and convolved with a $30^{\prime \prime}$ circular Gaussian beam.

description of the ISM that includes cold clouds in pressure equilibrium with hot, diffuse gas (e.g., McKee \& Ostriker 1977), and a prescription for star formation constrained by the Schmidt/Kennicutt laws (Kennicutt 1998; Schmidt 1959; see Springel \& Hernquist 2003). The BH(s) in the simulation are realized through sink particles that accrete gas from the surrounding ISM such that $0.5 \%$ of the accreted mass energy onto the central $\mathrm{BH}(\mathrm{s})$ is reinjected into the ISM as thermal energy (Di Matteo et al. 2005; Springel et al. 2005a, 2005b).

The progenitor disk galaxies used in this work are similar to the Milky Way, but with a higher gas fraction $(50 \%)$. Thus, they are likely representative of high- $z$ disk galaxies and possible progenitors of present-epoch ULIRGs. The methodology for constructing the model galaxies is given in Springel et al. (2005a). The progenitors utilized a softened equation of state (EOS) with softening parameter $q_{\mathrm{EOS}}=0.25$ (Springel et al. 2005a) such that the mass-weighted ISM temperature is $\sim 10^{4.5} \mathrm{~K}$. The galaxies had dark matter halos initialized to follow a Hernquist (1990) profile, and circular velocity $V_{200}=160 \mathrm{~km} \mathrm{~s}^{-1}$. The virial properties of the halos are scaled to be appropriate for $z=2$ (e.g., Robertson et al. 2006b) and follow the prescription given by Mo et al. (1998) for cosmological models of disk galaxies. The galaxies were set on a parabolic orbit with the orientation of the spin axis of each disk specified by the standard spherical coordinates, $\theta$ and $\phi$ (with $\theta_{1}=30^{\circ}, \phi_{1}=60^{\circ}, \theta_{2}=-30^{\circ}$, and $\phi_{2}=45^{\circ}$ ), and were initially separated by $140 \mathrm{kpc}$. We utilize 120,000 dark matter particles and 160,000 total disk particles, $50 \%$ of which represent gas, the rest serving as collisionless star particles. The gas, star, disk, and dark matter particle masses were $3.9 \times 10^{5}, 1.95 \times 10^{5}, 5.9 \times 10^{5}$, and $7.6 \times 10^{6} h^{-1} M_{\odot}$ each, respectively. The gravitational softening lengths were 100 $h^{-1}$ pc for baryons and $200 h^{-1}$ pc for dark matter particles. We have performed simulations with and without BHs. For the simulations with $\mathrm{BHs}$, the initial mass of the $\mathrm{BH}$ particle in each progenitor was $10^{5} h^{-1} M_{\odot}$, and the peak accretion rate was $\sim 0.5 M_{\odot}$ $\mathrm{yr}^{-1}$. The final mass of the remnant's BH is $\sim 5 \times 10^{7} h^{-1} M_{\odot}$.

In order to estimate molecular line emission, we have developed a three dimensional non-LTE radiative transfer code based on an improved version of the Bernes (1979) algorithm. Our improvements (described more fully in Narayanan et al. 2006 and D. Narayanan et al. 2006, in preparation) focus on including a subgrid model for giant molecular clouds (GMCs) in order to more accurately model the strongly density-dependent collisional excitation rates within our $\sim 10^{2}$ pc grid cells. We model GMCs as singular isothermal spheres (SISs) with 
power-law index 2 (Walker et al. 1990) and assume half the cold gas mass in each cell is bound in GMCs. Our results for higher lying molecular levels and high dipole moment molecules are particularly improved using this subgrid approach.

The emergent spectrum is built by integrating the equation of radiative transfer through numerous lines of sight (LOSs). The source functions in each grid cell are determined by the densities of molecules at levels $u$ and $l$ for a given transition $u \rightarrow l$. These level populations $n_{u}, n_{l}$ are dependent on the incident radiation field from other clouds; we thus guess at a solution, calculate the mean intensity field in a Monte Carlo manner, determine the updated level populations through the rate equations, and repeat the process until the populations have converged. In between each iteration, the code goes into a subresolution process in which it decomposes each cloud into an SIS and determines the level populations in each subresolution element. The non-LTE aspect of this treatment is particularly important as the assumption of LTE breaks down when considering the propagation of radiation through media with densities lower than the transition's critical density. For the radiative transfer calculations, we typically emitted $\sim 1 \times 10^{7}$ model photons per iteration and consider the $2.73 \mathrm{~K}$ microwave background as the boundary condition.

\section{RESULTS}

\subsection{Intensity Contour Maps}

In Figure 1 we show a series of snapshots in $\mathrm{CO} J=1-0$ intensity contour maps from the two merger simulations. The plot spans $45 h^{-1} \mathrm{Myr}$ and begins when the progenitors are approaching final coalescence. The $\mathrm{BH}$ accretion rate nears its peak as the BHs merge at $T \sim 1.15 h^{-1} \mathrm{Gyr}$, and thus the feedback energy input from the $\mathrm{AGN}$ is near its maximum in the model with BHs.

Beginning from $T \approx 1.13 h^{-1}$ Gyr onward, the CO morphology of the galaxy in the $\mathrm{BH}$ model undergoes dramatic changes owing to feedback from the buried AGN. Massive blobs of cold molecular gas entrained in the wind are visible through the $\mathrm{CO}$ $J=1-0$ tracer. Indeed, while the AGN wind may in detail evaporate cold clouds via thermal conduction, enough cold gas survives to be visible through molecular emission. Moreover, the clouds entrained in these outflows remain cold and dense enough to continue forming stars. The dense cores in these clouds emit at CO transitions with relatively high critical densities; consequently, the outflows are visible against the background at transitions as high as $\mathrm{CO} J=6-5$. In order to produce the observed emission comparable to that of the nucleus, the outflows must have large column densities. We find columns spanning the range $5 \times 10^{22} \mathrm{~cm}^{-2} \lesssim N\left(\mathrm{H}_{2}\right) \lesssim 1 \times 10^{23} \mathrm{~cm}^{-2}$ through the outflow in Figure 1, depending on the viewing angle (although we did not include a UV background in our models and thus the true column may be less). As the outflowing gas leaves the nuclear region at velocities of $\sim 200-300 \mathrm{~km} \mathrm{~s}^{-1}$, it becomes more diffuse, resulting in weaker $\mathrm{CO}$ emission. We find that the existence of molecular outflows is not unique to this particular model and is seen in other merger simulations that include BHs.

In the model without BHs (Fig. 1, right column), the molecular outflows on the $\sim \mathrm{kpc}$ scale observed in the BH model are not seen, highlighting the effects of AGN feedback in expelling loosely bound circumnuclear molecular gas. We note, however, that supernova-driven winds are not incorporated in these models. It is known, through absorption-line spectroscopy, that such winds in starbursts can induce outflows of comparable speeds (e.g., Heckman et al. 2000; Martin 2005; Rupke et al. 2005). While large columns of outflowing molecular gas have not been imaged in many of these systems, there are notable exceptions, such as the classic starburst M82 (Walter et al. 2002). We will explore the effects of supernova winds on the molecular gas in due course.

\subsection{Line Profiles}

The AGN-induced outflows also leave their imprint on spectral line profiles. In Figure 2 we have calculated the spectral line emission from $T=1.155 h^{-1} \mathrm{Gyr}$ in the model with BHs (Fig. 1, bottom left panel). The spectrum is generated along three orthogonal LOSs. In order to simulate an unresolved observation, we set the merger at $z=2\left(\Omega_{\Lambda}=0.7, \Omega_{M}=\right.$ $0.3, h=0.75)$ and convolved the emission from the $12 h^{-1}$ kpc image with a circular 30" ( 235 kpc) Gaussian beam.

Typically, in the $\mathrm{BH}$ model, once the galaxies have coalesced (when the BHs of the progenitors are indistinguishable in our simulations), the emission from the unresolved object is characteristic of a single Gaussian, centered at the systemic velocity of the galaxy. However, when viewing outflows with a strong LOS velocity component, a secondary peak appears superposed on the Gaussian emission line from the galaxy (e.g., Fig. 2, left panel); this peak is the emission from the outflow and is redshifted or blueshifted from line center at the LOS velocity of the outflow. While we have presented the CO $J=1-0$ emission, these line profiles are similar through CO $J=6-5$.

The emission peak corresponding to the outflow appears at the greatest offset velocity with respect to the systemic velocity of the galaxy when the outflow is moving mostly along the LOS (e.g., the $-\hat{z}$ observation; Fig. 2). If the observation is tilted such that a smaller component of the outflow velocity is along the LOS, the emission peak corresponding to the outflow will move closer to, and eventually will merge with, the broad emission peak of the galaxy (the $\hat{y}$ and $-\hat{x}$ observations, respectively, in Fig. 2). The peak temperature of the outflow emission may also decrease when the observation is tilted as the observer looks through less column. A given outflow along a particular LOS is typically visible via its line profile for an average of $\sim 10 h^{-1}$ Myr before the column density through the outflow drops such that its emission is no longer detectable against the broader Gaussian emission from the central region. We estimate the "outflow" profiles are visible $\sim 25 \%$ of the time in our simulations (which span $200 h^{-1} \mathrm{Myr}$ ), averaged over many viewing angles.

Double-peaked profiles have been observed in mergers that do not necessarily correspond to outflows; for example, observations of high-density gas in the prototypical ULIRG, Arp 220, have evidenced a symmetric double-peak profiled in which each peak corresponds to the starburst regions of nuclei of the progenitor galaxies (Taniguchi \& Shioya 1998; Sakamoto et al. 1999; Narayanan et al. 2005). Similar profiles also occur in systems in which there is significant rotation, i.e., a disk galaxy or rotating nuclear ring. However, some differences exist between the double peaks originating in progenitor galaxies or rotating systems and those caused by outflows. The double peaks characteristic of the former two cases are typically both broad and are symmetric about the systemic velocity of the galaxy. Conversely, the peak arising from the outflow is typically much narrower than the broad emission profile of the galaxy, due to its small velocity dispersion along the LOS. That said, the component of the profile from the outflow can be quite bright, owing to large $\mathrm{H}_{2}$ column densities through the outflowing material. 
The characteristic outflow profile consisting of a broad Gaussian with a narrow line superposed is also degenerate with that of high-velocity gas falling in toward the nucleus. The infalling clouds tend to have a higher velocity dispersion than clouds entrained in the AGN wind by, on average, $30 \%$, resulting in broader $\mathrm{CO}$ lines. It is unclear, however, from our simulations whether there is a significant enough difference between the velocity dispersion or column density in outflowing and infalling gas to determine the direction of flow from $\mathrm{CO}$ observations alone. There may be observational tests at other wavelengths to help break this degeneracy, however. In our simulations, infalling gas primarily leaves its imprint on the line profile just as the major merger has occurred, prior to the major AGN feedback phase. By serving as an indicator for AGN activity, X-ray observations may help distinguish the origin of the "outflow" line profile. For example, using simulations similar to those explored in this study, Cox et al. (2006) have shown that the X-ray luminosity from diffuse gas in galaxy mergers peaks during the phase of heavy $\mathrm{BH}$ accretion and that thermal energy input from AGN feedback can produce Xrays consistent with observations of ULIRGs with known embedded AGNs (e.g., UGC 5101; Imanishi et al. 2003). Elevated $\mathrm{X}$-ray emission, both from diffuse gas and from hard X-rays from the central BHs, may be indicative of a buried AGN. Correlations between hard X-ray flux and CO line profiles will be discussed in greater detail in D. Narayanan et al. (2006, in preparation).

At $z \approx 2$, where the infrared and submillimeter background are dominated by LIRGs and ULIRGs (Smail et al. 1997) and the quasar density is near its peak (e.g., Schneider et al. 2005), large molecular line surveys may prove fruitful in investigating the existence of a correspondence between double-peaked line profiles and quasar activity. Indeed, large fractions of submillimeter galaxies at high redshift show double-peaked line profiles ( 50\%; Greve et al. 2005; Tacconi et al. 2006), some of which appear to have line profiles similar to those presented in the leftmost and middle panels of Figure 2; similarly, large fractions $(\sim 75 \%)$ of submillimeter galaxies show AGN activity, as evidenced by IR and X-ray studies (Alexander et al. 2005; Polletta et al. 2006).

\section{SUMMARY AND CONCLUSIONS}

We have discussed two features of $\mathrm{CO}$ emission from cold molecular gas entrained in winds resulting from AGN feedback in galaxy mergers: (1) an extended CO morphology when the outflow is largely in the plane of the observation, and (2) kinematic features in the emission-line profile when the outflow has a significant LOS velocity component. There may, of course, be hybrid cases as well, in which features of both signatures of AGN feedback are detectable.

Observations of signatures such as these can help to interpret current observations of ULIRGs both at low and high $z$. Emission maps and line profiles similar to those presented in this work may have already been observed in local mergers (e.g., NGC 985; Appleton et al. 2002), as well as in $z \approx 2$ submillimeter selected sources (Greve et al. 2005; Tacconi et al. 2006). Upcoming surveys with high-resolution interferometers have the potential of constraining models of $\mathrm{BH}$ growth and associated AGN feedback in galaxy mergers.

D. N. acknowledges financial support from an NSF Graduate Research Fellowship.

\section{REFERENCES}

Alexander, D. M., Smail, I., Bauer, F. E., Chapman, S. C., Blain, A. W., Brandt, W. N., \& Ivison, R. J. 2005, Nature, 434, 738

Appleton, P., et al. 2002, ApJ, 566, 682

Barnes, J. E., \& Hernquist, L. E. 1991, ApJ, 370, L65 1996, ApJ, 471, 115

Bernes, C. 1979, A\&A, 73, 67

Bryant, P. M., \& Scoville, N. Z. 1999, AJ, 117, 2632

Cox, T. J., Di Matteo, T., Hernquist, L., Hopkins, P., Robertson, B., \& Springel, V. 2006, ApJ, in press

Davé, R., Hernquist, L., Katz, N., \& Weinberg, D. H. 1999, ApJ, 511, 521

Di Matteo, T., Springel, V., \& Hernquist, L. 2005, Nature, 433, 604

Farrah, D., Afonso, J., Efstathiou, A., Rowan-Robinson, M., Fox, M., \& Clements, D. 2003, MNRAS, 343, 585

Gao, Y., \& Solomon, P. M. 2004, ApJ, 606, 271

Greve, T. R., et al. 2005, MNRAS, 359, 1165

Heckman, T. M., Lehnert, M. D., Strickland, D. K., \& Armus, L. 2000, ApJS, 129,493

Hernquist, L. 1990, ApJ, 356, 359

Hopkins, P. F., Hernquist, L., Cox, T. J., Di Matteo, T., Martini, P., Robertson, B., \& Springel, V. 2005a, ApJ, 630, 705

Hopkins, P. F., et al. 2005b, ApJ, 625, L71

2005c, ApJ, 630, 716

2006a, ApJS, 163, 1

2006b, ApJS, 163, 50

Imanishi, M., Terashima, Y., Anabuki, N., \& Nakagawa, T. 2003, ApJ, 596, L167

Iono, D., et al. 2004, ApJ, 616, L63

Kennicutt, R. C., Jr. 1998, ApJ, 498, 541

Lynden-Bell, D. 1969, Nature, 223, 690

Martin, C. L. 2005, ApJ, 621, 227

McKee, C. F., \& Ostriker, J. P. 1977, ApJ, 218, 148

Mihos, J. C., \& Hernquist, L. E. 1996, ApJ, 464, 641

Mo, H. J., Mao, S., \& White, S. D. M. 1998, MNRAS, 295, 319
Narayanan, D., Groppi, C. E., Kulesa, C. A., \& Walker, C. K. 2005, ApJ, 630, 269

Narayanan, D., Kulesa, C. A., Boss, A. P., \& Walker, C. K. 2006, ApJ, in press

Polletta, M., et al. 2006, ApJ, 642, in press (astro-ph/0602228)

Robertson, B., Cox, T. J., Hernquist, L., Franx, M., Hopkins, P. F., Martini, P., \& Springel, V. 2006a, ApJ, 641, 21

Robertson, B., Hernquist, L., Cox, T. J., Di Matteo, T., Hopkins, P. F., Martini, P., \& Springel, V. 2006b, ApJ, 641, 90

Rupke, D., Veilleux, S., \& Sanders, D. B. 2005a, ApJS, 160, 87

Sakamoto, K, et al. 1999, ApJ, 514, 68

Sanders, D. B., \& Mirabel, I. F. 1996, ARA\&A, 34, 749

Sanders, D. B., Soifer, B. T., Elias, J. H., Madore, B. F., Matthews, K., Neugebauer, G., \& Scoville, N. Z. 1988a, ApJ, 325, 74

Sanders, D. B., Soifer, B. T., Elias, J. H., Neugebauer, G., \& Matthews, K. 1988b, ApJ, 328, L35 1991, ApJ, 370, 158

Schmidt, M. 1959, ApJ, 129, 243

Schneider, D. P., et al. 2005, AJ, 130, 367

Smail, I., Ivison, R. J., \& Blain, A. W. 1997, ApJ, 490, L5

Soifer, B. T., et al. 1987, ApJ, 320, 238

Springel, V. 2005, MNRAS, 364, 1105

Springel, V., Di Matteo, T., \& Hernquist, L. 2005a, MNRAS, 361, 776 2005b, ApJ, 620, L79

Springel, V., \& Hernquist, L. 2002, MNRAS, 333, 649 2003, MNRAS, 339, 312

Tacconi, L. J., et al. 2006, ApJ, 640, 228

Taniguchi, Y., \& Shioya, Y. 1998, ApJ, 501, L167

Walker, C. K., Adams, F. C., \& Lada, C. J. 1990, ApJ, 349, 515

Walter, F., Weiss, A., \& Scoville, N. 2002, ApJ, 580, L21

Wang, J., Zhang, Q., Wang, Z., Ho, P. T. P., Fazio, G., \& Wu, Y. 2004, ApJ, 616, L67 